\title{
Numerical meshless solution of high-dimensional sine-Gordon equations via Fourier HDMR-HC approximation
}

\author{
Xin $\mathrm{Xu}$ - Xiaopeng Luo · Herschel Rabitz
}

\begin{abstract}
In this paper, an implicit time stepping meshless scheme is proposed to find the numerical solution of high-dimensional sine-Gordon equations (SGEs) by combining the high dimensional model representation (HDMR) and the Fourier hyperbolic cross (HC) approximation. To ensure the sparseness of the relevant coefficient matrices of the implicit time stepping scheme, the whole domain is first divided into a set of subdomains, and the relevant derivatives in high-dimension can be separately approximated by the Fourier HDMR-HC approximation in each subdomain. The proposed method allows for stable large time-steps and a relatively small number of nodes with satisfactory accuracy. The numerical examples show that the proposed method is very attractive for simulating the high-dimensional SGEs.
\end{abstract}

Keywords Sine-Gordon equations · Meshless methods · High dimensional model representation

\section{Introduction}

The sine-Gordon equation (SGE) is a nonlinear hyperbolic partial differential equation (PDE) involving the d'Alembert operator and the sine of the unknown function, and the SGE plays an important role in many mathematical physics applications. It was originally introduced by Bour [6] and rediscovered by Frenkel and

\footnotetext{
$\mathrm{X} . \mathrm{Xu}$

Department of Chemistry, Princeton University, Princeton, NJ 08544, USA

School of Managment and Engineering, Nanjing University, Nanjing, 210008, China

E-mail: xuxin103@163.com,xx2@princeton.edu

X. Luo

Department of Chemistry, Princeton University, Princeton, NJ 08544, USA

School of Managment and Engineering, Nanjing University, Nanjing, 210008, China

E-mail: luo_works@163.com,xiaopeng@princeton.edu

H. Rabitz

Department of Chemistry, Princeton University, Princeton, NJ 08544, USA

Program in Applied and Computational Mathematics, Princeton University, Princeton, NJ 08544, USA

E-mail: hrabitz@princeton.edu
} 
Kontorova [23]. Further details about the background and applications of the SGE can be found in $[1,19,57,62]$. In this paper, we focus on developing an effective means for the numerical solution of the SGE in an arbitrary number of dimensions. A $(n+1)$-dimensional SGE generally takes the form:

$$
\begin{aligned}
& u_{t t}(\boldsymbol{x}, t)+\beta u_{t}(\boldsymbol{x}, t)=\Delta u(\boldsymbol{x}, t)-\psi(\boldsymbol{x}) \sin (u(\boldsymbol{x}, t)), \\
& \boldsymbol{x}=\left(x_{1}, x_{2}, \cdots, x_{n}\right) \in \Omega, t>0,
\end{aligned}
$$

where $n$ is a positive integer, $\Delta$ is the Laplacian operator in $n$ spatial dimensions, $\Omega=\left[a_{1}, b_{1}\right] \times\left[a_{2}, b_{2}\right] \times \cdots \times\left[a_{n}, b_{n}\right] \subseteq \mathbb{R}^{n}$. The initial conditions associated with Eq.(1) are given by

$$
\begin{gathered}
u(\boldsymbol{x}, 0)=v_{1}(\boldsymbol{x}), \quad \boldsymbol{x} \in \Omega \\
u_{t}(\boldsymbol{x}, 0)=v_{2}(\boldsymbol{x}), \boldsymbol{x} \in \Omega,
\end{gathered}
$$

and the Neumann boundary conditions are

$$
\frac{\partial u}{\partial \boldsymbol{l}}(\boldsymbol{x}, t)=w(\boldsymbol{x}, t), \boldsymbol{x} \in \Gamma, t>0
$$

where $\boldsymbol{l}$ denotes the (typically exterior) normal to the boundary of the domain, and $\Gamma$ is the boundary of $\Omega$, i.e $\Gamma=\partial \Omega$. The real parameter $\beta \geqslant 0$ weights the dissipative term. When $\beta=0$, Eq.(1) reduces to an undamped SGE in $n$ spatial variables, while when $\beta>0$, the damped SGE is obtained. The function $\psi(\boldsymbol{x})$ can be interpreted as a Josephson current density, while $v_{1}$ and $v_{2}$ in Eqs.(2) and (3) represent wave modes or the kink and velocity, respectively.

The $(1+1)$-dimensional SGE first appeared in a strictly mathematical context in differential geometry regarding the theory of surfaces of constant curvature [38]. Moreover, it is well known that the $(1+1)$-dimensional SGE arises in many important systems, such as the Thirring model, the Coulomb gas system and the ferromagnetic $X Y$ model, etc. [45, 46, 51]. Because of its wide applications, the $(1+1)$-dimensional SGE has been studied with a variety of numerical methods, including finite difference methods (FDM) and finite element methods (FEM), etc. [2]. Recently, additional solution methods have been proposed including collocation $[15,37]$, the boundary integral approach [13, 11], and a combination of the finite difference with the the diagonally implicit Runge-Kutta-Nyström (DIRKN) method [49], etc.

There is recent interest in the SGE in higher dimensions. As Barone et al. [5] pointed out, Eq.(1) also has been applied in many branches of physics for the $n=2$ and 3 cases. The exact solutions for the undamped SGE in higher dimensions have been obtained by Hirota's method [29], Lamb's method [66], the Bäcklund transformation [10] and Painlevé transcendents [34], etc. Moreover, numerical solutions for the $(2+1)$-dimensional undamped SGE have been proposed by Christiansen and Lomdahl [9] using a generalized leapfrog method, Guo et al. [28] using two finite difference schemes, Argyris et al. [3] using finite elements. Xin [64] studied the SGE as an asymptotic reduction of the two level dissipationless Maxwell-Bloch system, Sheng et al. [59] presented a numerical method with a split cosine scheme, and Bratsos [7] used a three-time level fourth-order explicit finite difference scheme to solve the undamped SGE. Following a similar approach, Bratsos [8] transformed the SGE to a second-order initial value problem with the help of the method of lines. Numerical approaches for the damped SGE were proposed by Nakajima et al. 
[50] who considered dimensionless loss factors and unitless normalized bias, and Gorria et al. [24] investigated nonlinear wave propagation in a planar wave guide consisting of two rectangular regions joined by a bent domain of constant curvature using as a model of the kink solution to the SGE. Additionally, Dehghan and Mirzaei [14] developed the dual reciprocity boundary element method for both the undamped and damped $(2+1)$-dimensional SGE, and Jiwari et al. [33] obtained a numerical scheme based on a polynomial differential quadrature method.

Although the SGE is nonintegrable except for $n=1$, some properties and exact solutions for the $(n+1)$-dimensional SGE have been obtained by various methods. Kobayashi and Izutsu [36] extensively studied the exact traveling wave solutions of the SGE in the field of theoretical physics. Many additional mathematical methods have been proposed for finding traveling wave solutions of the SGE. Feng [21] applied the Painlevé analysis to the study of an approximate SGE and its traveling solitary wave solution in $(n+1)$-dimensional space. With the help of exact solutions to the cubic nonlinear Klein-Gordon fields, Lou et al. [41] studied the exact solutions for the $(n+1)$-dimensional SGE. Adopting $\beta=0$ and $\psi(\boldsymbol{x})=1$, de la Hoz and Vadillo [30] generalized the exact soliton solution for the $(n+1)$-dimensional SGE:

$$
u(\boldsymbol{x}, t)=4 \arctan \left[C \exp \left(\sum_{i=1}^{n} a_{i} x_{i}-b t\right)\right]
$$

where $\sum_{i=1}^{n} a_{i}^{2}=1+b^{2}$. By adopting the proper ansatz, more general solutions can be obtained for the multi-dimensional SGE, including the three-dimensional case allowing for non-constant $C$ [1]. Obtaining the exact solution for the general SGE would be ideal, but unfortunately it is very difficult for practical engineering problems that are usually complex in nature. Despite numerical methods commonly used in many types of linear and nonlinear PDEs, de la Hoz and Vadillo [30] remarked that there were no references to the numerical treatment of the SGE for dimensions larger than three, which motivated them to propose a numerical method for the $n$-dimensional SGE based on using operational matrices.

Many standard numerical methods for solving PDEs are widely used in engineering, but they usually require the construction and update of a mesh, which is an inherent disadvantage. In order to overcome these difficulties, recently the meshless numerical method has attracted attention. This method can establish a system of algebraic equations over the entire problem domain without using a predefined mesh. Rather, a set of scattered nodes, called field nodes, are used within the problem domain as well as on the boundaries of the domain [40]. The meshless method does not require a priori information about the relationship between the nodes for the interpolation or approximation of unknown functions over the field of variables [40]. Employing the meshless method, Dehghan and Shokri [15] studied the one-dimensional nonlinear SGE and used Thin Plate Spline Radial Basis Functions (TPS-RBF) to approximate the solution, and they also applied the TPS-RBF method to both the Klein-Gordon equation [17] and the two-dimensional SGE [16]. A series of meshless approaches have been presented $[4,12,31,32,35,47,48,52,58,62]$. Moreover, since the nodal distribution for most existing meshless methods is preassigned, Xu et al. [65] proposed a numerical two-step meshless method for soliton-like structures based on the optimal sampling density of kernel interpolation. 
In dealing with high-dimensional PDEs, obtaining good quality approximate solutions is a difficult problem because of the so-called 'curse of dimensionality'. High dimensional model representation (HDMR) [39, 54, 55, 56, 60] provides a viable approach based on the fact that high-dimensional functions often can be efficiently expressed as sums of low-dimensional functions. The HDMR decomposition is also well known in statistics as the ANOVA (analysis of variance) decomposition [20, 22, 25, 61]. In recent years, the HDMR decomposition has been under rapid development becoming an important tool for understanding highdimensional functions $[25,26,27,39,42,43,54,55,56,60]$. In this paper, we will use a HDMR decomposition in conjunction with the Fourier hyperbolic cross (HC) approximation [44].

The remainder of the paper is organized as follows: Section 2 presents a function approximation method using HDMR-HC. Then a new meshless numerical scheme is proposed in Section 3 for solving the $(n+1)$-dimensional SGE using the HDMRHC approximation. In Section 4, we provide several examples with a comparative numerical error analysis. Section 5 summarizes the relevant results.

\section{Function approximation using HDMR-HC and the partition of unity}

\subsection{HDMR-HC approximation}

Let $n \geqslant 2, \lambda \in \mathbb{R}_{+}$and $f(\boldsymbol{x}) \in \mathcal{W}_{1}^{s}\left(\mathbb{T}_{\lambda}^{n}\right), s \in \mathbb{N}_{0}$ be a $n$-variate function which is $\frac{1}{\lambda}$-periodic in each variable, where $\mathbb{T}_{\lambda}^{n}$ is the $n$-torus given by [44]

$$
\mathbb{T}_{\lambda}^{n}:=\left[-\frac{1}{2 \lambda}, \frac{1}{2 \lambda}\right)^{n}
$$

and the function space $\mathcal{W}_{1}^{s}\left(\mathbb{T}_{\lambda}^{n}\right)$ is defined by

$$
\mathcal{W}_{1}^{s}\left(\mathbb{T}_{\lambda}^{n}\right)=\left\{f \in \mathcal{L}\left(\mathbb{T}_{\lambda}^{n}\right): \partial^{r} f \in \mathcal{L}\left(\mathbb{T}_{\lambda}^{n}\right),|r|_{\infty} \leqslant s\right\}
$$

with the norm

$$
\|f\|_{\mathcal{W}_{1}^{s}\left(\mathbb{T}_{\lambda}^{n}\right)}=\sum_{0 \leqslant|r|_{\infty} \leqslant s}\left\|\partial^{r} f\right\|_{\mathcal{L}\left(\mathbb{T}_{\lambda}^{n}\right)}
$$

where $r \in \mathbb{N}_{0}^{n}$ denotes a $n$-dimensional multi-index with the norm

$$
|r|_{\infty}:=\max _{1 \leqslant j \leqslant n} r_{j}
$$

and the $r$-th order mixed derivative is given by

$$
\partial^{r} f:=\frac{\partial^{|r|_{1}} f}{\partial x_{1}^{r_{1}} \cdots \partial x_{n}^{r_{n}}}
$$

We consider the multivariate Fourier series of $f(\boldsymbol{x})$

$$
f(\boldsymbol{x}) \sim \sum_{m \in \mathbb{Z}^{n}} c_{m} \mathrm{e}^{2 \pi \mathrm{i} \lambda m \cdot \boldsymbol{x}}, \boldsymbol{x} \in \mathbb{T}_{\lambda}^{n}
$$

where the Fourier coefficients $c_{m}$ are defined by

$$
c_{m}=c_{m}(f)=\lambda^{n} \int_{\mathbb{T}_{\lambda}^{n}} f(\boldsymbol{x}) \mathrm{e}^{-2 \pi \mathrm{i} \lambda m \cdot \boldsymbol{x}} \mathrm{d} \boldsymbol{x}, m \in \mathbb{Z}^{n} .
$$


For a nonempty set $\left\{j_{1}, \cdots, j_{v}\right\} \subset\{1, \cdots, n\}$, let

$$
\begin{aligned}
\Lambda_{j_{1}, \cdots, j_{v}}:= & \left\{m \in \mathbb{Z}^{n}: m_{l} \neq 0 \forall l \in\left\{j_{1}, \cdots, j_{v}\right\}\right. \\
& \text { and } \left.m_{l}=0 \forall l \notin\left\{j_{1}, \cdots, j_{v}\right\}, l \in\{1, \cdots, n\}\right\}
\end{aligned}
$$

and

$$
\Lambda_{j_{1}, \cdots, j_{v}}^{k_{\lambda}}:=\left\{m \in \Lambda_{j_{1}, \cdots, j_{v}}: k_{\lambda} \leqslant(2 \pi)^{v} \lambda_{j_{1}} \cdots \lambda_{j_{v}}\left|m_{j_{1}} \cdots m_{j_{v}}\right|<k_{\lambda}+1, k_{\lambda} \in \mathbb{N}\right\} .
$$

Note that $\Lambda_{0}$ is the set consisting of the $n$-dimensional zero vector. Then $\mathbb{Z}^{n}$ can be decomposed into the following form

$$
\mathbb{Z}^{n}=\Lambda_{0}+\sum_{j} \Lambda_{j}+\sum_{j_{1}<j_{2}} \Lambda_{j_{1}, j_{2}}+\cdots+\Lambda_{j_{1}, \cdots, j_{n}}
$$

and we refer to this as a HDMR decomposition of $\mathbb{Z}^{n}$; then a multiple Fourier series can be decomposed with an HDMR structure

$$
\begin{aligned}
\sum_{m \in \mathbb{Z}^{n}} c_{m} \mathrm{e}^{2 \pi i \lambda m \cdot x}= & c_{0}+\sum_{k_{\lambda}=1}^{\infty}\left(\sum_{j} \sum_{m \in \Lambda_{j}^{k_{\lambda}}} c_{m} \mathrm{e}^{2 \pi i \lambda m \cdot x}\right. \\
& \left.+\sum_{j_{1}<j_{2}} \sum_{m \in \Lambda_{j_{1}, j_{2}}^{k_{\lambda}}} c_{m} \mathrm{e}^{2 \pi i \lambda m \cdot x}+\cdots+\sum_{m \in \Lambda_{j_{1}, \cdots, j_{n}}^{k_{\lambda}}} c_{m} \mathrm{e}^{2 \pi i \lambda m \cdot x}\right)
\end{aligned}
$$

If let

$$
\Lambda^{K_{\lambda}}=\Lambda_{0}+\sum_{k_{\lambda}=1}^{K_{\lambda}}\left(\sum_{j} \Lambda_{j}^{k_{\lambda}}+\sum_{j_{1}<j_{2}} \Lambda_{j_{1}, j_{2}}^{k_{\lambda}}+\cdots+\Lambda_{j_{1}, \cdots, j_{d}}^{k_{\lambda}}\right),
$$

that is,

$$
\Lambda^{K_{\lambda}}:=\left\{m \in \mathbb{Z}^{n}: \prod_{j=1}^{n} \max \left\{2 \pi \lambda\left|m_{j}\right|, 1\right\} \leqslant K_{\lambda}\right\},
$$

then we define the Fourier HDMR-HC partial sum up to $K_{\lambda}$-th order [44]

$$
S_{K_{\lambda}}(\boldsymbol{x}, f)=\sum_{m \in \Lambda^{K_{\lambda}}} c_{m} \mathrm{e}^{2 \pi \mathrm{i} \lambda m \cdot \boldsymbol{x}}, \boldsymbol{x} \in \mathbb{T}_{\lambda}^{n} .
$$

Suppose $M$ is the number of points $\boldsymbol{x} \in \mathbb{T}_{\lambda}^{n}$, which depends on both $\lambda$ and $n$, and

$$
F_{\lambda, m}(\boldsymbol{x})=\mathrm{e}^{2 \pi \mathrm{i} \lambda m \cdot \boldsymbol{x}}
$$

then Eq.(14) can be rewritten as

$$
S_{K_{\lambda}}(\boldsymbol{x}, f)=\sum_{m=1}^{M} c_{m} F_{\lambda, m}(\boldsymbol{x}), \boldsymbol{x} \in \mathbb{T}_{\lambda}^{n}
$$

Moreover, it follows that, for any $f \in \mathcal{W}_{1}^{s}\left(\mathbb{T}_{\lambda}^{n}\right), s>\gamma+p+1$ and $K_{\lambda} \geqslant \delta$, the bound

$$
\left|\partial^{r} S_{K_{\lambda}}(\boldsymbol{x}, f)-\partial^{r} f(\boldsymbol{x})\right| \leqslant C_{n, \lambda, s, p, \gamma, \delta}\|f\|_{\mathcal{W}_{1}^{s}\left(\mathbb{T}_{\lambda}^{n}\right)} K_{\lambda}^{-(s-\gamma-p-1)}, \quad|r|_{\infty}=p
$$


holds pointwise almost everywhere, where the constant $C_{n, \lambda, s, p, \gamma, \delta}$ depends on $n, \lambda, s, p$ and $\delta$.

From the definition of $\mathbb{T}_{\lambda}^{n}$, we find that $\mathbb{T}_{\lambda}^{n}$ is smaller as $\lambda$ becomes larger. The convergence rate of the Fourier HDMR-HC partial sums $S_{K_{\lambda}}$ is closely related to the value of $\lambda$. In particular, for a given accuracy, when $\lambda$ is large, $S_{K_{\lambda}}$ generally is well approximated by a low order truncated HDMR, and this is the basic starting point of the Fourier HDMR-HC approximation. For example, if there is a function of $n=5$ variables, and suppose $\lambda=1 / \pi$ and the desired accuracy is $\varepsilon=\mathbf{O}\left(K_{\lambda}^{-(s-p-\gamma-1)}\right)$, then a $K_{\lambda}$-th order Fourier HDMR-HC partial sum $S_{K_{\lambda}}$ is just a 2 nd order truncated HDMR of the function when $K_{\lambda} \leqslant 31$. Therefore, we expect that a low order truncated HDMR-HC can be used to effectively capture the behavior of a high-dimensional function and its derivatives.

\subsection{Partition of unity}

In this subsection, we will discuss how to approximate a function via HDMR-HC. Suppose $u(\boldsymbol{x})$ is a function defined on $\mathbb{R}$, where $\boldsymbol{x} \in \Omega \subseteq \mathbb{R}^{n}$. First, we divide the domain $\Omega$ into $D \in \mathbb{N}$ subdomains, denoted as $\Omega_{j},(j=1,2, \cdots, D)$, thus $\Omega=\bigcup_{j=1}^{D} \Omega_{j}$. In each subdomain $\Omega_{j}$, let $\boldsymbol{x}_{j}$ be the centre of the region, and let $\chi_{j}$ denote all $M_{j}$ nodes in $\Omega_{j}$, i.e., $\sum_{j=1}^{D} M_{j}=N$. Further, suppose $u(\boldsymbol{x}), \boldsymbol{x} \in \Omega_{j}$ can be represented as $u_{j}$, then we have

$$
u_{j}(\boldsymbol{x})=J_{\lambda}\left(\boldsymbol{x}-\boldsymbol{x}_{j}\right) u(\boldsymbol{x})=J_{j} u(\boldsymbol{x}), \boldsymbol{x} \in \Omega_{j}
$$

where

$$
\Omega_{j} \subset \operatorname{supp} J_{\lambda}\left(\boldsymbol{x}-\boldsymbol{x}_{j}\right) \text { and } \operatorname{supp} J_{\lambda}(\boldsymbol{x}) \subset \mathbb{T}_{\lambda}^{n}
$$

is the characteristic function of $u_{j}$ satisfying

$$
\sum_{j=1}^{D} J_{j}=1, \forall \boldsymbol{x} \in \Omega \subseteq \mathbb{R}^{n}
$$

From Eq.(15), $u_{j}$ at any point $\boldsymbol{x} \in \Omega_{j}$ can be approximated as

$$
\hat{u}_{j}(\boldsymbol{x})=\sum_{m_{j}=1}^{M_{j}} c_{m_{j}} F_{\lambda, m_{j}}\left(\boldsymbol{x}-\boldsymbol{x}_{j}\right)=\boldsymbol{F}_{\boldsymbol{\lambda}}^{\mathrm{T}}(\boldsymbol{x}) \boldsymbol{c}_{j}
$$

where $\boldsymbol{F}_{\boldsymbol{\lambda}}^{\mathrm{T}}(\boldsymbol{x})=\left[F_{\lambda, 1}, F_{\lambda, 2}, \cdots, F_{\lambda, M_{j}}\right]$ with the same $\lambda$ for all nodes and $c_{m_{j}}$ is the $m_{j}$-th unknown coefficient. Utilizing the values of these $M_{j}$ nodes, there are $M_{j}$ equations with one for each node, then we have following matrix form

$$
\boldsymbol{U}_{j}=\boldsymbol{F}_{j} \boldsymbol{c}_{j}
$$

where

$$
\boldsymbol{U}_{j}=\left[u_{1}, u_{2}, \cdots, u_{M_{j}}\right]^{\mathrm{T}}
$$

is the vector of function values at the $M_{j}$ nodes, and

$$
\boldsymbol{c}_{j}=\left[c_{1}, c_{2}, \cdots, c_{M_{j}}\right]^{\mathrm{T}}
$$


is the vector of undetermined coefficients with

$$
\boldsymbol{F}_{j}=\left[\begin{array}{cccc}
F_{\lambda, 11} & F_{\lambda, 12} & \cdots & F_{\lambda, 1 M_{j}} \\
F_{\lambda, 21} & F_{\lambda, 22} & \cdots & F_{\lambda, 2 M_{j}} \\
\vdots & \vdots & & \vdots \\
F_{\lambda, M_{j} 1} & F_{\lambda, M_{j} 2} & \cdots & F_{\lambda, M_{j} M_{j}}
\end{array}\right]
$$

where $F_{\lambda, i m_{j}}=F_{\lambda, m_{j}}\left(\boldsymbol{\chi}_{i}\right)$. Suppose $\boldsymbol{F}_{j}^{-1}$ exists (i.e., this condition can always be satisfied [53,63]), then $\boldsymbol{c}_{j}$ can be obtained by solving Eq.(20), i.e.

$$
\boldsymbol{c}_{j}=\boldsymbol{F}_{j}^{-1} \boldsymbol{U}_{j}
$$

From Eq.(16), we have

$$
U_{j}=J_{j} \boldsymbol{U}
$$

where $\boldsymbol{U}=\bigcup_{j=1}^{D} \boldsymbol{U}_{j}$. Then

$$
\boldsymbol{c}_{j}=\boldsymbol{F}_{j}^{-1} J_{j} \boldsymbol{U}
$$

Substitute both Eq.(21) and Eq.(22) back into Eq.(19), we have an approximation of function $u_{j}$ :

$$
\hat{u}_{j}(\boldsymbol{x})=\boldsymbol{F}_{\boldsymbol{\lambda}}^{\mathrm{T}}(\boldsymbol{x}) \boldsymbol{F}_{j}^{-1} J_{j} \boldsymbol{U}
$$

Furthermore, from Eqs.(16) and (18) we have

$$
u(\boldsymbol{x})=\sum_{j=1}^{D} J_{j} u(\boldsymbol{x})=\sum_{j=1}^{D} u_{j}(\boldsymbol{x}), \boldsymbol{x} \in \Omega
$$

Then $u(\boldsymbol{x})$ at any point $\boldsymbol{x} \in \Omega$ can be formally approximated as

$$
\begin{aligned}
\hat{u}(\boldsymbol{x}) & =\sum_{j=1}^{D} \boldsymbol{F}_{\boldsymbol{\lambda}_{j}^{\mathrm{T}}}^{\mathrm{T}}(\boldsymbol{x}) \boldsymbol{F}_{j}^{-1} J_{j} \boldsymbol{U} \\
& =\sum_{j=1}^{D} J_{j} \boldsymbol{F}_{\boldsymbol{\lambda}}^{\mathrm{T}}(\boldsymbol{x}) \boldsymbol{F}_{j}^{-1} \boldsymbol{U} \\
& =\boldsymbol{\Phi}^{\mathrm{T}}(\boldsymbol{x}) \boldsymbol{U}
\end{aligned}
$$

\section{Solution for $(n+1)$-dimensional SGEs}

Now, we present the numerical scheme for solving the $(n+1)$-dimensional SGE based on using the Fourier HDMR-HC approximation. Suppose the approximated function of the field function $u(\boldsymbol{x}),\left(\boldsymbol{x}=\left\{x_{1}, x_{2}, \cdots, x_{n}\right\}\right)$ is formally denoted as

$$
\hat{u}(\boldsymbol{x})=\sum_{m=1}^{M_{j}} c_{m_{j}} F_{\lambda, m_{j}}\left(\boldsymbol{x}-\boldsymbol{x}_{j}\right)=\boldsymbol{F}_{\boldsymbol{\lambda}}^{\mathrm{T}}(\boldsymbol{x}) \boldsymbol{F}_{j}^{-1} \boldsymbol{U}_{\boldsymbol{j}}
$$

where $M_{j}$ is the number of field nodes used in the selected domain, and $\boldsymbol{U}_{\boldsymbol{j}}$ is the vector that collects the true nodal function values for these $M_{j}$ field nodes, and $\boldsymbol{x}_{j}$ 
is the centre of this selected region. Further, the derivatives of $u(\boldsymbol{x})$ at any point $\boldsymbol{x}$ can be approximated as

$$
\frac{\partial^{p}}{\partial x_{l}^{p}} \hat{u}(\boldsymbol{x})=\frac{\partial^{p} \boldsymbol{F}_{\boldsymbol{\lambda}}^{\mathrm{T}}(\boldsymbol{x})}{\partial x_{l}^{p}} \boldsymbol{F}_{j}^{-1} \boldsymbol{U}_{\boldsymbol{j}}
$$

where $x_{l}$ denotes one element of $\boldsymbol{x}=\left\{x_{1}, x_{2}, \cdots, x_{n}\right\}$.

In this paper, the time derivatives are approximated by the time-stepping method and we have the following approximation:

$$
\begin{gathered}
\frac{\partial^{2} u}{\partial t^{2}} \approx \frac{1}{\tau^{2}}\left[u^{(k+1)}-2 u^{(k)}+u^{(k-1)}\right] \\
\frac{\partial u}{\partial t} \approx \frac{1}{2 \tau}\left[u^{(k+1)}-u^{(k-1)}\right]
\end{gathered}
$$

where $\tau$ is the time step, and $u^{(k)}$ is the approximate value of $u(\boldsymbol{x}, t)$ at $\left(\boldsymbol{x}, t_{k}\right)$, $t_{k}=k \tau$. Moreover the Crank-Nicolson scheme is used to approximate $u$ at three respective times as

$$
u(\boldsymbol{x}, t) \approx \frac{1}{3}\left[u^{(k+1)}+u^{(k)}+u^{(k-1)}\right]
$$

To manage the nonlinearity, a Quasilinearization Method (QLM) is adopted. The QLM is very effective for dealing with the nonlinear aspects of the SGE and other PDEs. In this fashion the nonlinear term in Eq. (1) can be represented as

$$
\sin (u)=\sin \left(u^{(k)}\right)+\left(u^{(k+1)}-u^{(k)}\right) \cos \left(u^{(k)}\right)
$$

Thus, Eq.(1) can be discretized as

$$
\begin{aligned}
& \frac{1}{3} \Delta u^{(k+1)}-\left(\eta+\mu+\varphi^{(k)}\right) u^{(k+1)} \\
= & -\frac{1}{3} \Delta u^{(k)}-\left(2 \mu+\varphi^{(k)}\right) u^{(k)}+\psi(\boldsymbol{x}) \sin \left(u^{(k)}\right) \\
& -\frac{1}{3} \Delta u^{(k-1)}-(\eta-\mu) u^{(k-1)}
\end{aligned}
$$

where $\mu=\tau^{-2}, \eta=\frac{\beta}{2 \tau}$ and $\varphi^{(k)}=\psi(\boldsymbol{x}) \cos \left(u^{(k)}\right)$.

Suppose $N$ field nodes are denoted as $\chi=\{\chi\}_{1}^{N}$, where $N$ is determined by both $D$ and $M_{j}$. Then from Eq.(26) we have an approximation of the field variable $u$ according to the HDMR-HC approximation

$$
\hat{u}(\boldsymbol{x})=\boldsymbol{\Phi}^{\mathrm{T}}(\boldsymbol{x}) \boldsymbol{U}=\sum_{j=1}^{N} \phi_{j}(\boldsymbol{x}) u_{j}
$$

where $\phi_{j}(\boldsymbol{x})$ depends on $J_{j}, \boldsymbol{F}_{\boldsymbol{\lambda}}^{\mathrm{T}}(\boldsymbol{x})$ and $\boldsymbol{F}_{j}^{-1}$. The derivatives of $\hat{u}$ can be approximated as

$$
\Delta \hat{u}(\boldsymbol{x})=\Delta \boldsymbol{\Phi}^{\mathrm{T}}(\boldsymbol{x}) \boldsymbol{U}
$$


Therefore, for any point $\boldsymbol{x}_{i}$, the approximation in Eq.(33) can be written as

$$
\begin{aligned}
& \sum_{j=1}^{N}\left[\frac{1}{3} \Delta \phi_{j}\left(\boldsymbol{x}_{i}\right)-\left(\eta+\mu+\varphi^{(k)}\right) \phi_{j}\left(\boldsymbol{x}_{i}\right)\right] u_{j}^{(k+1)} \\
= & \sum_{j=1}^{N}\left[-\frac{1}{3} \Delta \phi_{j}\left(\boldsymbol{x}_{i}\right)-\left(2 \mu+\varphi^{(k)}\right) \phi_{j}\left(\boldsymbol{x}_{i}\right)\right] u_{j}^{(k)} \\
& +\sum_{j=1}^{N}\left[-\frac{1}{3} \Delta \phi_{j}\left(\boldsymbol{x}_{i}\right)-(\eta-\mu) \phi_{j}\left(\boldsymbol{x}_{i}\right)\right] u_{j}^{(k-1)} \\
& +\psi\left(\boldsymbol{x}_{i}\right) \sin \left(u^{(k)}\right)
\end{aligned}
$$

Let $A_{i j}=\frac{1}{3} \Delta \phi_{j}\left(\boldsymbol{x}_{i}\right), B_{i j}=\phi_{j}\left(\boldsymbol{x}_{i}\right)$, and $E_{i j}^{(k)}=A_{i j}-\left(\eta+\mu+\varphi^{(k)}\right) B_{i j}, G_{i j}^{(k)}=$ $-A_{i j}-\left(2 \mu+\varphi^{(k)}\right) B_{i j}, H_{i j}=A_{i j}-(\eta-\mu) B_{i j}, C_{i}^{(k)}=\psi\left(\boldsymbol{x}_{i}\right) \sin \left(u^{(k)}\right)$, then Eq.(36) can be re-written as

$$
\sum_{j=1}^{N} E_{i j}^{(k)} u_{j}^{(k+1)}=\sum_{j=1}^{N} G_{i j}^{(k)} u_{j}^{(k)}+\sum_{j=1}^{N} H_{i j} u_{j}^{(k-1)}+C_{i}^{(k)}
$$

For all $N$ field nodes $\chi$ we have following matrix form:

$$
\boldsymbol{E}^{\prime(k)} \hat{\boldsymbol{u}}^{(k+1)}=\boldsymbol{G}^{\prime(k)} \hat{\boldsymbol{u}}^{(k)}+\boldsymbol{H}^{\prime} \hat{\boldsymbol{u}}^{\prime(k-1)}+\boldsymbol{C}^{\prime(k)}
$$

where $\boldsymbol{C}^{\prime(k)}=\left[C_{1}^{(k)}, C_{2}^{(k)}, \cdots, C_{N}^{(k)}\right]^{\mathrm{T}}$.

In using a meshless strong method to solve the PDE, the solution can be unstable if there is a derivative boundary condition, so the fictitious points method is used to impose derivative boundary conditions [40]. Suppose there are $N_{b}$ nodes on the boundary, then along the derivative boundaries, another $N_{b}$ fictitious points are added outside of the domain. Two sets of equations are established at each derivative boundary node: one for the derivative boundary condition, and the other for the governing equation. With the $N_{b}$ additional degrees of freedom, $\left\{u_{(N+1)}, u_{(N+2)}, \cdots, u_{\left(N+N_{b}\right)}\right\}$, added into the system, then Eq.(36) can be rewritten as

$$
\sum_{j=1}^{N+N_{b}} E_{i j}^{(k)} u_{j}^{(k+1)}=\sum_{j=1}^{N+N_{b}} G_{i j}^{(k)} u_{j}^{(k)}+\sum_{j=1}^{N+N_{b}} H_{i j} u_{j}^{(k-1)}+C_{i}^{(k)}
$$

and for a node at $\boldsymbol{x}_{i_{b}}$, that is, on the derivative boundary, the derivative boundary conditions have the form

$$
\sum_{j=1}^{N+N_{b}} \frac{\partial}{\partial l} \phi_{j}\left(\boldsymbol{x}_{i_{b}}\right) u_{j}^{(k+1)}=-\sum_{j=1}^{N+N_{b}} \frac{\partial}{\partial l} \phi_{j}\left(\boldsymbol{x}_{i_{b}}\right) u_{j}^{(k)}-\sum_{j=1}^{N+N_{b}} \frac{\partial}{\partial l} \phi_{j}\left(\boldsymbol{x}_{i_{b}}\right)^{(k-1)}+3 w\left(\boldsymbol{x}_{i_{b}}, t\right)
$$

Assembling Eqs.(39) and (40) for the corresponding nodes, the discretized global system equation becomes

$$
\boldsymbol{E}^{(k)} \hat{\boldsymbol{u}}^{(k+1)}=\boldsymbol{G}^{(k)} \hat{\boldsymbol{u}}^{(k)}+\boldsymbol{H} \hat{\boldsymbol{u}}^{(k-1)}+\boldsymbol{C}^{(k)}
$$


where $\boldsymbol{E}, \boldsymbol{G}, \boldsymbol{B}$ and $\boldsymbol{H}$ are $\left(N+N_{b}\right) \times\left(N+N_{b}\right)$ matrices, $\hat{\boldsymbol{u}}=\left[\hat{u}_{1}, \hat{u}_{2}, \cdots, \hat{u}_{\left(N+N_{b}\right)}\right]^{\mathrm{T}}$, $\boldsymbol{C}^{(k)}=\left[C_{1}^{(k)}, C_{2}^{(k)}, \cdots, C_{N}^{(k)}, 3 w\left(\boldsymbol{x}_{N+1}, t\right), 3 w\left(\boldsymbol{x}_{N+2}, t\right), \cdots, 3 w\left(\boldsymbol{x}_{N+N_{b}}, t\right)\right]^{\mathrm{T}}$. At the first time level, i.e. $k=0$, we adopt the following:

$$
\hat{\boldsymbol{u}}^{(0)}=\boldsymbol{v}_{\mathbf{1}}
$$

and

$$
\hat{\boldsymbol{u}}^{(-1)}=\hat{\boldsymbol{u}}^{(1)}-2 \tau \boldsymbol{v}_{\mathbf{2}}
$$

where $\boldsymbol{v}_{\mathbf{1}}$ and $\boldsymbol{v}_{\mathbf{2}}$ are the initial conditions for all nodes $\boldsymbol{\chi}$ introduced in Eqs.(2) and (3).

\section{Numerical experiments}

In this section, the proposed meshless numerical scheme is applied to several examples to show the efficiency and accuracy for the $(n+1)$-dimensional SGE. As mentioned in the previous section, to approximate the time derivatives we use a finite difference method, so an iterative scheme is employed to reach the final time $t$. In order to test the performance of the numerical solution, we use the $L_{\infty}$ error and root-mean-square (RMS) error norms defined as

$$
L_{\infty}=\left\|f\left(x_{i}\right)-\hat{f}\left(x_{i}\right)\right\|_{\infty}=\max _{1 \leqslant i \leqslant N}\left|f\left(x_{i}\right)-\hat{f}\left(x_{i}\right)\right|
$$

and

$$
\operatorname{RMS}=\sqrt{\frac{1}{N} \sum_{i=1}^{N}\left|f\left(x_{i}\right)-\hat{f}\left(x_{i}\right)\right|^{2}}
$$

where $N$ is the number of nodes, $f\left(x_{i}\right)$ is the exact solution, and $\hat{f}\left(x_{i}\right)$ is the numerical solution. To assess both the stability and the solution accuracy, we compute the condition number of the system matrix, which is defined as

$$
\kappa(\boldsymbol{F})=\|\boldsymbol{F}\|\left\|\boldsymbol{F}^{-1}\right\|
$$

where $\kappa(\boldsymbol{F})$ depends on the parameter $\lambda$ and the number of nodes $N$.

4.1 Test problem for a $(2+1)$-dimensional SGE

The test problem for a $(2+1)$-dimensional SGE has the following form $[18,16,33]$

$$
\begin{array}{r}
u_{t t}=\Delta u-\sin (u), \\
(x, y) \in \Omega, t>0,
\end{array}
$$

where $\Omega=[-7,7] \times[-7,7]$, and the initial conditions are

$$
\begin{aligned}
& v_{1}(x, y)=4 \tan ^{-1}[\exp (x+y)], \quad(x, y) \in \Omega \\
& v_{2}(x, y)=-\frac{4 \exp (x+y)}{1+\exp (2 x+2 y)}, \quad(x, y) \in \Omega .
\end{aligned}
$$


and the Neumann boundary condition is

$$
w(x, y, t)=\frac{4 \exp (x+y-t)}{1+\exp (2 x+2 y-2 t)},(x, y) \in \partial \Omega, t>0
$$

The analytic solution of this problem is:

$$
u(x, y, t)=4 \tan ^{-1}[\exp (x+y-t)],(x, y) \in \Omega
$$

In this example, both the proposed HDMR-HC meshless method and the radial basis function (RBF) method in [16] are used to numerically solve the equation. Since the field nodes of the RBF method in [16] is the Sobol sequence with $N=$ 3249 , then we adopt the same total number of field nodes for the HDMR-HC meshless method $(D=49)$. The time step $\tau$ is set to 0.001 . The results of the two different measures of error are presented in Table 1. We see that with the same number of field nodes, the errors of the proposed HDMR-HC meshless method are smaller than those of the RBF method. The condition numbers at particular times are also listed in Table 1.

Table 1 Errors and condition number $\kappa$ for $(2+1)$-D SGE

\begin{tabular}{cccccc}
\hline \multirow{2}{*}{ Time $(s)$} & \multicolumn{2}{c}{$L_{\infty}$-error } & \multicolumn{2}{c}{ RMS-error } & \multirow{2}{*}{ RBF } \\
& HDMR-HC & RBF & HDMR-HC & \\
\hline 1.0 & 0.0670 & 0.0326 & 0.0050 & 0.0043 & $3.4 \times 10^{5}$ \\
3.0 & 0.0834 & 0.0343 & 0.0103 & 0.0045 & $5.0 \times 10^{5}$ \\
5.0 & 0.1015 & 0.0355 & 0.0145 & 0.0045 & $4.2 \times 10^{5}$ \\
7.0 & 0.1516 & 0.0368 & 0.0187 & 0.0047 & $6.3 \times 10^{5}$ \\
\hline
\end{tabular}

Note: The results of RBF method come from Ref. [16]

4.2 Test problem for a $(5+1)$-dimensional SGE

To further test the proposed HDMR-HC scheme, we choose a $(5+1)$-dimensional example, which involves all the implementation issues explained in the previous subsection. The exact solution has the form of Eq.(5)

$$
u(\boldsymbol{x}, t)=4 \arctan \left[C \exp \left(\sum_{i=1}^{5} a_{i} x_{i}-b t\right)\right], \boldsymbol{x}=\left(x_{1}, x_{2}, \cdots, x_{5}\right) \in \Omega, t>0
$$

where $C=1, b=1, \Omega=[-6,6] \times[-6,6] \times \cdots \times[-6,6] \subseteq \mathbb{R}^{5}$ and

$$
a_{i}=\left\{\begin{array}{c}
\sqrt{2} / 2, i=1,2,3 \\
1 / 2, \quad i=4,5
\end{array}\right.
$$

The initial conditions are

$$
\begin{aligned}
& v_{1}(\boldsymbol{x})=4 \arctan \left[C \exp \left(\sum_{i=1}^{5} a_{i} x_{i}\right)\right], \boldsymbol{x} \in \Omega, \\
& v_{2}(\boldsymbol{x})=-\frac{4 b C \exp \left(\sum_{i=1}^{n} a_{i} x_{i}\right)}{1+C^{2} \exp \left(\sum_{i=1}^{n} 2 a_{i} x_{i}\right)}, \boldsymbol{x} \in \Omega,
\end{aligned}
$$


and the Neumann boundary conditions are

$$
w_{j}(\boldsymbol{x}, t)=\frac{4 a_{j} C \exp \left(\sum_{i=1}^{5} a_{i} x_{i}-b t\right)}{1+C^{2} \exp \left(\sum_{i=1}^{5} 2 a_{i} x_{i}-2 b t\right)}, j=1,2, \cdots, 5, \boldsymbol{x} \in \partial \Omega, t>0
$$

Both the proposed HDMR-HC method and the RBF method are used to solve the equation. The Sobol sequence is chosen as the field nodes with a total number of $N=2^{16}$ for the HDMR-HC method ( $D=243$ and we use the HDMR approximation up to order 3 ) and $N^{\prime}=2^{18}$ for the RBF method. In this case the time step is chosen as $\tau=0.2$. Table 2 presents $L_{\infty}$, RMS errors and the condition number $\kappa$ at some selected times $t$.

Table 2 Errors and condition number $\kappa$ for $(5+1)$-D SGE

\begin{tabular}{cccccc}
\hline \multirow{2}{*}{ Time $(s)$} & \multicolumn{2}{c}{$L_{\infty}$-error } & \multicolumn{2}{c}{ RMS-error } & \multirow{2}{*}{ RBF } \\
& HDMR-HC & RBF & HDMR-HC & \\
\hline 1.0 & 0.2071 & 0.1083 & 0.0130 & 0.0076 & $4.4 \times 10^{6}$ \\
3.0 & 0.1956 & 0.0910 & 0.0143 & 0.0079 & $3.7 \times 10^{6}$ \\
5.0 & 0.2132 & 0.1205 & 0.0165 & 0.0080 & $4.5 \times 10^{6}$ \\
7.0 & 0.2203 & 0.1124 & 0.0187 & 0.0081 & $4.9 \times 10^{6}$ \\
\hline
\end{tabular}

\section{Conclusions}

In this paper, we propose a new meshless solution method for high-dimensional sine-Gordon equations. First, we present a function approximation using the HDMR$\mathrm{HC}$ decomposition. Then we divided the whole domain into several subdomains with the help of the partition of unity, and obtain a function approximation at any random point in each subdomain. Hence, we develop a numerical procedure for the high-dimensional SGEs by a meshless strong solution method. The time-stepping method is used to approximate the time derivatives of SGEs, and a quasilinearization scheme is performed to treat the nonlinearity of the equation. Finally, to demonstrate the accuracy of the proposed method with two numerical experiments. The examples suggest that the proposed procedure is attractive for solving high-dimensional SGEs.

Acknowledgements The authors X.X. and X.L. acknowledge support from the National Science Foundation (Grant No. CHE-1763198), and H.R. acknowledges support from the Templeton Foundation (Grant No. 52265).

\section{References}

1. Aero E, Bulygin A, Pavlov Y (2009) Solutions of the three-dimensional sineGordon equation. Theoretical and Mathematical Physics 158:313-319 
2. Argyris J, Haase M (1987) An engineer's guide to soliton phenomena, Application of the finite element method. Computer Methods in Applied Mechanics and Engineering 61:71-122

3. Argyris J, Haase M, Heinrich J (1991) Finite element approximation to twodimensional sine-Gordon solitons. Computer Methods in Applied Mechanics and Engineering 86:1-26

4. Asgari Z, Hosseini S (2013) Numerical solution of two-dimensional sine-Gordon and MBE models using Fourier spectral and high order explicit time stepping methods. Computer Physics Communications 184:565-572

5. Barone A, Esposito F, Magee C, Scott A (1971) Theory and applications of the sine-Gordon equation. La Rivista del Nuovo Cimento 1(2):227-267

6. Bour E (1862) Théorie de la déformation des surfaces. J Ecole Imperiale Polytechnique 19:1-48

7. Bratsos A (2005) An explicit numerical scheme for the sine-Gordon equation in $2+1$ dimensions. Appl Numer Anal Comput Math 2:189-211

8. Bratsos A (2007) The solution of the two-dimensional sine-Gordon equation using the method of lines. Journal of Computational and Applied Mathematics 206:251-277

9. Christiansen P, Lomdahl P (1981) Numercal solution of $2+1$ dimensional sine-Gordon solitons. Physica D 2:482-494

10. Christiansen P, Olsen O (1979) On dynamical two-dimensional solutions to the sine-Gordon equation. Z Angew Math Mech 56:30-31

11. Dehghan M, Ghesmati A (2010) Application of the dual reciprocity boundary integral equation technique to solve the nonlinear Klein-Gordon equation. Computer Physics Communications 181(8):1410-1418

12. Dehghan M, Ghesmati A (2010) Numerical simulation of two-dimensional sinegordon solitons via a local weak meshless technique based on the radial point interpolation method (RPIM). Computer Physics Communications 181:772786

13. Dehghan M, Mirzaei D (2008) The boundary integral equation approach for numerical solution of the one-dimensional sine-Gordon equation. Numerical Methods for Partial Differential Equations 24:1405-1415

14. Dehghan M, Mirzaei D (2008) The dual reciprocity boundary element method (DRBEM) for two-dimensional sine-Gordon equation. Computer Methods in Applied Mechanics and Engineering 197:476-486

15. Dehghan M, Shokri A (2008) A numerical method for one-dimensional nonlinear sine-Gordon equation collocation and radial basis functions. Numerical Methods for Partial Differential Equations 24:687-698

16. Dehghan M, Shokri A (2008) A numerical method for solution of the twodimensional sine-Gordon equation using the radial basis functions. Mathematics and Computers in Simulation 79(3):700-715

17. Dehghan M, Shokri A (2009) Numerical solution of the nonlinear Klein-Gordon equation using radial basis functions. Journal of Computational and Applied Mathematics 230(2):400-410

18. Djidjeli K, Price W, Twizell E (1995) Numerical solutions of a damped sineGordon equation in two space variables. Journal of Engineering Mathmatics 29:347-369

19. Drazin P, Johnson R (1989) Solitons: An Introduction. Cambridge University Press 
20. Efron B, Stein C (1981) The jackknife estimate of variance. The Annals of Statistics 9(3):586-596

21. Feng Z (2004) An approximate sine-Gordon equation and its traveling wave solution in $(n+1)$-dimensional space. Applied Mathematics and Computation 152:597-610

22. Fisher R (1925) Statistical methods for research workers. Genesis Publishing Pvt Ltd

23. Frenkel J, Kontorova T (1939) On the theory of plastic deformation and twinning. Journal of Physics (USSR) 1:137-149

24. Gorria C, Gaididei Y, Soerensen M, Christiansen P, Caputo J (2004) Kink propagation and trapping in a two-dimensional curved Josephson junction. Physical Review B 69:1-10

25. Griebel M, Holtz M (2010) Dimension-wise integration of high-dimensional functions with applications to finance. Journal of Complexity 26:455-489

26. Griebel M, Kuo F, Sloan I (2010) The smoothing effect of the ANOVA decomposition. Journal of Complexity 26:523-551

27. Griebel M, Kuo F, Sloan I (2013) The smoothing effect of integration in $\mathbb{R}^{d}$ and the ANOVA decomposition. Mathematics of Computation 82(281):383-400

28. Guo B, Rodriguez P, Vazquez L (1986) Numerical solution of the sine-Gordon equation. Applied Mathematics and Computation 18:1-14

29. Hirota R (1973) Exact three-soliton solution of the two-dimensional sineGordon equation. Journal of the Physical Society of Japan 35:15-66

30. de la Hoz F, Vadillo F (2012) Numerical simulation of the $n$-dimensional sineGordon equation via operational matrices. Computer Physics Communications 183:864-879

31. Jiang Z, Wang R (2012) Numerical solution of one-dimensional Sine-Gordon equation using high accuracy multiquadric quasi-interpolation. Applied Mathematics and Computation 218(15):7711-7716

32. Jiwari R, Pandit S, Mittal R (2012) A differential quadrature algorithm to solve the two dimensional linear hyperbolic telegraph equation with Dirichlet and Neumann boundary conditions. Applied Mathematics and Computation 218(13):7279-7294

33. Jiwari R, Pandit S, Mittal R (2012) Numerical simulation of two-dimensional sine-Gordon solitons by differential quadrature method. Computer Physics Communications 183:600-616

34. Kaliappan P, Lakshmanan M (1979) Kadomtsev-Petviashvili and twodimensional sine-Gordon equations: reduction to Painlevé transcendents. Phys A: Math Gen 249:1-23

35. Karamanli A, Mugan A (2013) Strong form meshless implementation of taylor series method. Applied Math ematics and Computati on 219:9069-9080

36. Kobayashi K, Izutsu M (1976) Exact solution on the $n$-dimensional sineGordon equation. Journal of the Physical Society of Japan 41(3):1091-1092

37. Lakestani M, Dehghan M (2010) Collocation and finite difference-collocation methods for the solution of nonlinear Klein-Gordon equation. Computer Physics Communications 181(8):1392-1401

38. Lamb G (1971) Analytical descriptions of ultrashort optical pulse propagation in a resonant medium. Reviews of Modern Physics 43:99-124

39. Li G, Rosenthal C, Rabitz H (2001) High dimensional model representations. The Journal of Physical Chemistry A 105(33):7765-7777 
40. Liu G, Gu Y (2005) An Introduction to Meshfree Methods and Their Programing. Springer

41. Lou S, Hu H, Tang X (2005) Interactions among periodic waves and solitary waves of the $(n+1)$-dimensional sine-gordon field. Physical Review E 71(3):036604

42. Luo X, Lu Z, Xu X (2014) Non-parametric kernel estimation for the ANOVA decomposition and sensitivity analysis. Reliability Engineering and System Safety 130:140-148

43. Luo X, Lu Z, Xu X (2014) Reproducing kernel technique for high dimensional model representations (HDMR). Computer Physics Communications 185(12):3099-3108

44. Luo X, Xu X, Rabitz H (2017) On the fundamental conjecture of HDMR: a Fourier analysis approach. Journal of Mathematical Chemistry 55:632-660

45. Minnhagen P (1985) Nonuniversal jumps and the Kosterlitz-Thouless transition. Physical Review Letters 54(21):2351-2354

46. Minnhagen P (1987) The two-dimensional Coulomb gas, vortex unbinding, and superfluid-superconducting films. Review of Modern Physics 59:1001-1066

47. Mirzaei D, Dehghan M (2009) Implementation of meshless LBIE method to the 2D non-linear SG problem. International Journal for Numerical Methods in Engineering 79(13):1662-1682

48. Mirzaei D, Dehghan M (2010) Meshless local Petrov-Galerkin (MLPG) approximation to the two dimensional sine-Gordon equation. Journal of Computational and Applied Mathematics 233(10):2737-2754

49. Mohebbi A, Dehghan M (2010) High-order solution of one-dimensional sineGordon equation using compact finite difference and DIRKN methods. Mathematical and Computer Modelling 51:537-549

50. Nakajima K, Onodera Y, Nakamura T, Sato R (1974) Numerical analysis of vortex motion on Josephson structures. Journal of Applied Physics 45:40954099

51. Ni G, Lou S, Chen S, Lee H (1990) Two-dimensional Coulomb gas studied in the sine-Gordon formulation. Physical Review B: Condensed Matter 41(10):6947-6957

52. Pekmen B, Tezer-Sezgin M (2012) Differential quadrature solution of nonlinear Klein" cGordon and sine-Gordon equations. Computer Physics Communications 183(8):1702-1713

53. Powell M (1992) Advanced in Numerical Analysis, Oxford Univercity Press, chap Theory of radial basis function approximation in 1990, pp 303-322

54. Rabitz H, Alis Ö (1999) General foundations of high-dimensional model representations. Journal of Mathematical Chemistry 25(2-3):197-233

55. Rabitz H, Alis Ö (2000) Sensitivity analysis, John Wiley and Sons Publishers, chap Managing the tyranny of parameters in mathematical modeling of physical systems, pp 199-223

56. Rabitz H, Alis Ö, Shorter J, Shim K (1998) Efficient input-output model representations. Computer Physics Communications 117(1-2):11-20

57. Scott A, Chu F, McLaughlin D (1973) The soliton: A new concept in applied science. Proceedings of the IEEE 61(10):1443-1483

58. Shao W, Wu X (2014) The numerical solution of the nonlinear Klein-Gordon and Sine-Gordon equations using the Chebyshev tau meshless method. Computer Physics Communications 185:1399-1409 
59. Sheng Q, Khaliq A, Voss D (2005) Numerical simulation of two-dimensional sine-Gordon solitons via a split cosine scheme. Mathematics and Computers in Simulation 68:355-373

60. Sobol I (1993) Sensitivity estimates for nonlinear mathematical models. Mathematical Modeling and Computational Experiment 1(4):407-414, english translation

61. Stone C (1994) The use of polynomial splines and their tensor products in multivariate function estimation. The Annals of Statistics 22(1):118-171

62. Taleei A, Dehghan M (2014) A pseudo-spectral method that uses an overlapping multidomain technique for the numerical solution of sine-Gordon equation in one and two spatial dimensions. Mathematical Methods in the Applied Sciences 37(13):1909-1923

63. Wendland H (1998) Error estimates for interpolation by compactly supported radial basis functions of minimal degree. J Approx Theory 93:258-396

64. Xin J (2000) Modeling light bullets with the two-dimensional sine-Gordon equation. Physica D 135:345-368

65. Xu X, Lu Z, Luo X (2015) A numerical meshless method of soliton-like structures model via an optimal sampling density based kernel interpolation. Computer Physics Communications 192:12-22

66. Zagrodzinsky J (1979) Particular solutions of the sine-gordon equation in $2+1$ dimensions. Physics Letters A 72:287-286 